\title{
Der Bundestag zwischen Wahl und Entsendung zu seinem Präsidium: die Causa Bisky
}

\author{
Sebastian Lovens
}

Die Geschäftsordnung des Deutschen Bundestages (GO-BT) unterliegt als zentrales Regelungsinstrument des parlamentarischen Miteinanders gleichermaßen den konkreten Anforderungen des jeweiligen Bundestages als auch dem Anspruch, den Parlamentariern ${ }^{1}$ gleichberechtigten Zugang zu den innerparlamentarischen Mitwirkungs- und Gestaltungsrechten zu vermitteln. Dies gilt - mittelbar - auch für die als Fraktionen verfassten Zusammenschlüsse von Abgeordneten. Durch Bekanntmachung vom 26. September 20062 änderte der 16. Bundestag das in $\$ 2$ GO-BT enthaltene Wahlverfahren zu seinem Präsidium und regelte erstmalig das weitere Vorgehen für den Fall, dass ein Einzelbewerber für die Wahl zum Bundestagspräsidium in den ersten beiden Wahlgängen nicht die erforderliche Mehrheit erreicht. Dieser Änderung war die mehrmalige Nichtwahl des ersten Kandidaten der Fraktion Die Linke für das Amt des Bundestagsvizepräsidenten, Lothar Bisky, vorausgegangen.

\section{Der Anlass: ein zunächst unbesetztes Amt}

Die Wahl des Präsidiums des 16. Bundestages evozierte eine politische und parlamentsrechtliche Problemlage: Das mit dem Beginn der 13. Wahlperiode in der Geschäftsordnung formulierte Ziel, dass jede Fraktion mit mindestens einem Vizepräsidenten im Leitungsgremium des Parlaments vertreten ist, wurde zunächst verfehlt. Ein halbes Jahr lang - bis zur Wahl von Petra Pau am 7. April 2006 - blieb die Position eines Vizepräsidenten unbesetzt. Der Abgeordnete Bisky erreichte in den ersten vier Wahlgängen für die von der Fraktion Die Linke vorzuschlagende Vizepräsidentenposition nicht die jeweils erforderliche Mehrheit. Die Wahlen zum Präsidium im unmittelbaren zeitlichen Zusammenhang mit der Konstituierung des Bundestages erbrachten folgende Ergebnisse (vgl. Tabelle 1). ${ }^{3}$

$\$ 2$ Abs. 2 Satz 1 GO-BT in der zu Beginn der 16. Wahlperiode geltenden Fassung ${ }^{4}$ bestimmt für die ersten beiden Wahlgänge in Anlehnung an Art. 121 GG die Mehrheit der Mitglieder des Bundestages als erforderliches Quorum für die Wahl zu dessen Präsidium.

1 Die durchgängige Verwendung der männlichen Sprachform dient allein der besseren Lesbarkeit.

2 BGBl. I, S. 2210. Die Geschäftsordnung in dieser Fassung wird im Weiteren als GO-BT 2006 bezeichnet.

3 Die Ergebnisse von Wahlen zu früheren Bundestagspräsidien sind Rupert Schick, Die Bundestagspräsidenten, 16. Auflage, München 2003, S. $231 \mathrm{ff}$. zu entnehmen.

4 Der 16. Bundestag hat in seiner konstituierenden Sitzung am 18. Oktober 2005 die Weitergeltung der Geschäftsordnung des 15. Deutschen Bundestages beschlossen, vgl. das Protokoll 6 A. Am Ende der 15. Legislaturperiode galt die Geschäftsordnung des Deutschen Bundestages in der Fassung der Bekanntmachung vom 2. Juli 1980, BGBl. I, S. 1237, zuletzt geändert laut Bekanntmachung vom 12. Juli 2005, BGBl. I, S. 2512. Diese Fassung der Geschäftsordnung wird im Weiteren als GO-BT 2005 bezeichnet. Die spätere Änderung ließ $\$ 2$ Abs. 2 Satz 1 unverändert. 


\begin{tabular}{|c|c|c|c|c|c|}
\hline \multicolumn{6}{|c|}{ Tabelle 1: Wablen zum Präsidium des 16. Deutschen Bundestages } \\
\hline Name / Stimmen & abgegeben & gültig & ja & nein & $\begin{array}{l}\text { Enthal- } \\
\text { tungen }\end{array}$ \\
\hline $\begin{array}{l}\text { Präsident Norbert Lammert (CDU/ } \\
\text { CSU) }\end{array}$ & 607 & 606 & 564 & 25 & 17 \\
\hline Gerda Hasselfeldt (CDU/CSU) & 605 & 604 & 510 & 47 & 47 \\
\hline Wolfgang Thierse (SPD) & 605 & 605 & 417 & 136 & 52 \\
\hline Susanne Kastner $(\mathrm{SPD})^{\mathrm{a}}$ & 600 & 599 & 496 & 61 & 42 \\
\hline Hermann Otto Solms (FDP) & 602 & 602 & 486 & 85 & 31 \\
\hline $\begin{array}{l}\text { Lothar Bisky (Die Linke) } \\
\text { (1. Wahlgang) }\end{array}$ & 594 & 592 & 225 & 312 & 55 \\
\hline $\begin{array}{l}\text { Katrin Göring-Eckardt } \\
\text { (Bündnis 90/Die Grünen) }\end{array}$ & 587 & 587 & 479 & 69 & 39 \\
\hline $\begin{array}{l}\text { Lothar Bisky (Die Linke) } \\
\text { (2. Wahlgang) }\end{array}$ & 572 & 563 & 282 & 235 & 46 \\
\hline $\begin{array}{l}\text { Lothar Bisky (Die Linke) } \\
\text { (3. Wahlgang) }\end{array}$ & 544 & 537 & 248 & 258 & 31 \\
\hline $\begin{array}{l}\text { Lothar Bisky (Die Linke) } \\
\text { (4. Wahlgang) }^{\mathrm{b}}\end{array}$ & 595 & 595 & 249 & 310 & 36 \\
\hline \multicolumn{6}{|c|}{$\begin{array}{l}\text { a Dass die zweitstärkste Fraktion, die der SPD, zwei Vizepräsidenten stellt, ist mit den Stimmen der } \\
\text { Fraktionen von CDU/CSU und SPD und gegen die Fraktionen der FDP, der Linken und Bündnis } \\
\text { 90/Die Grünen beschlossen worden, vgl. Protokoll, a.a.O., } 10 \text { A. } \\
\text { b Der vierte Wahlgang fand in der zweiten Sitzung des 16. Deutschen Bundestages am 8. November } \\
2005 \text { statt. } \\
\text { Quelle: Protokoll der 1. Sitzung des } 16 \text {. Deutschen Bundestages vom 18. Oktober 2005, } 3 \text { D ff. und } \\
\text { das Protokoll der 16. Sitzung des Deutschen Bundestages vom } 8 \text {. November 2005, } 41 \text { B f. }\end{array}$} \\
\hline
\end{tabular}

Somit ist im ersten und zweiten Wahlgang die Kanzlermehrheit erforderlich. ${ }^{5}$ Unter Berücksichtigung der Überhangmandate betrug die gesetzliche Mitgliederzahl $614^{6}$, das Quorum lag somit bei 308. Mit Ausnahme von Lothar Bisky erreichten alle Kandidaten zur Wahl des Präsidiums das Quorum im ersten Wahlgang. Bisky erhielt auch im zweiten Wahl-

5 Historisch wechselte das erforderliche Quorum: Die GO der Nationalversammlung vom 6. Februar 1919 erforderte wie die preußischen Vorgängerregelungen die absolute Mehrheit, die GO des Reichstages vom 12. Dezember 1922 ließ die Mehrheit der abgegebenen Stimmen ausreichen - ebenso die vorläufige GO-BT vom 20. September 1949. Die Stimmen der Mehrheit der Mitglieder des Bundestages, also die Kanzlermehrheit, erfordert die GO-BT seit ihrer ersten endgültigen Fassung vom 6. Dezember 1951. Vgl. Otto Th. L. Zschucke, Die Geschäftsordnungen der deutschen Parlamente, Berlin 1928, S. 22 und passim, sowie Deutscher Bundestag (Hrsg.), Die Geschäftsordnungen deutscher Parlamente seit 1848, Bonn 1986, zu $₫ 2$. Zum geltenden Recht vgl. Joseph Bücker, Präsident und Präsidium, in: Hans-Peter Schneider / Wolfgang Zeh (Hrsg.), Parlamentsrecht und Parlamentspraxis, Berlin / New York 1989, \$27 Rn. 21; Hans Troßmann, Parlamentsrecht des Deutschen Bundestages, Kommentierung GO-BT, München 1977, $\$ 2$ Rn. 2.3.

6 Siehe http://www.bundestag.de/parlament/wahlen/sitzverteilung/1541_16.html (letzter Seitenaufruf 24. Oktober 2007). Der Abgeordnete Matthias Wissmann (CDU) schied am 1. Juni 2007 aus dem Bundestag aus. Da zu Gunsten der Landesliste des Bundeslandes, in dem dieser ein Direktmandat errang, Überhangmandate bestanden, wurde der Sitz nicht nachbesetzt, die gesetzliche Mitgliederzahl des Deutschen Bundestages beträgt seit dem 1. Juni 2007 somit 613. 
gang nicht die erforderliche Mehrheit. Weitere Wahlgänge und deren Quoren für den Fall eines Einzelkandidaten sah die GO-BT 2005 nicht vor. ${ }^{7}$ Der frühere Vize- und nunmehrige Präsident Norbert Lammert reagierte darauf zunächst in der konstituierenden und sodann in der zweiten Sitzung des Bundestages mit dem Vorschlag je eines weiteren Wahlgangs mit der relativen Mehrheit als Quorum, das heißt, für eine Wahl sollten lediglich mehr Ja- als Nein-Stimmen ohne Gewichtung der Enthaltungen ausreichen. ${ }^{8}$ Er begründete dies mit dem „Gesamtkontext der Regelungen“ zur Wahl des Bundestagspräsidiums ${ }^{9}$ ersichtlich darum bemüht, die Hürden im Wahlverfahren abzusenken, um die Zielvorgabe der Präsenz aller Fraktionen im Präsidium noch zu erreichen. Das Plenum schloss sich dem Verfahren eindeutig, aber nicht einstimmig an. ${ }^{10}$ Dennoch wurde Bisky auch nach diesem Modus nicht gewählt: Anders als im zweiten Wahlgang erhielt er auch im dritten und im vierten Wahlgang nicht die relative Mehrheit. Weitere Wahlgänge mit Bisky als Kandidaten fanden nicht statt. Seine Fraktion sah nach den Fehlschlägen im Herbst 2005 auch „bis auf weiteres" davon ab, einen anderen Kandidaten für das Amt vorzuschlagen. ${ }^{11}$ Das für die viertgrößte Fraktion im Bundestag vorgehaltene Vizepräsidentenamt blieb somit zunächst unbesetzt. Erstmalig wieder seit den ersten beiden Wahlperioden mit Fraktionen der Grünen im Parlament - der 10. und der 11. - waren nicht alle Fraktionen im Bundestagspräsidium vertreten. ${ }^{12}$ Jedoch konnten sich die Grünen damals noch nicht auf die Vorgabe in der GO-BT berufen, jede Fraktion sei im Präsidium vertreten, denn diese existiert erst seit der 13. Wahlperiode. ${ }^{13}$

Umso dramatischer stellten sich das Ergebnis und die daran anknüpfende Eigendynamik der missglückten Kandidatur Biskys dar: Mit der Übernahme der GO der Vorgängerperiode drückte der 16. Bundestag seinen Wunsch aus, dass jede Fraktion in seinem Präsidium vertreten sein solle. Der konkreten Umsetzung dieses Ziels verweigerten sich die Abgeordneten jedoch zunächst. Die Motivationen werden mannigfaltig und individuell gewesen sein, von der Person Biskys selbst über seine Zugehörigkeit zur Linkspartei bis hin zu seiner Eigenschaft als deren Parteivorsitzender. Auch mag die kompromisslose Haltung der Fraktion Die Linke, an dem mehrfach erfolglosen Kandidaten festzuhalten, den einen oder anderen Abgeordneten bewogen haben, ein Zeichen der Freiheit seines Mandats zu setzen - es ist bemerkenswert, dass die Zustimmung ab dem zweiten Wahlgang kontinuierlich ab-

$7 \$ 2$ Abs. 2 Satz 3 GO-BT 2005 sah lediglich eine Stichwahl zwischen zwei Bewerbern vor, die beide das erforderliche Quorum nicht erreicht hätten.

$8 \mathrm{Zu}$ Verfahren und Organen der Geschäftsordnungsauslegung siehe Thomas Schwerin, Der Deutsche Bundestag als Geschäftsordnungsgeber, Berlin 1998, S. 255 ff.

9 Vgl. Protokoll, a.a.O., 14 B.

10 Vgl. ebenda und Protokoll der 2. Sitzung des 16. Deutschen Bundestages vom 8. November $2005,41 \mathrm{~B} \mathrm{f}$.

11 Zwar gab es bereits Ende Januar Überlegungen in der Fraktion, einen weiteren Wahlgang mit einer Kandidatin anzustreben, die Fraktion sah jedoch vor, diese sogar intern erst nach Mai 2006 zu diskutieren, vgl. die tageszeitung vom 1. Februar 2006.

12 In den Anfangsjahren der Republik waren bis zu acht Fraktionen im Parlament vertreten, die nicht alle berücksichtigt wurden. Seit der Endphase der 3. Wahlperiode gab es bis zum Einzug der Grünen in der 10. Wahlperiode konstant drei Fraktionen, die alle im Präsidium vertreten waren. Die Grünen hatten in der 10. und 11. Wahlperiode keinen Sitz im Präsidium, seit der 12. Wahlperiode waren alle Fraktionen repräsentiert; vgl. die Übersicht bei Rupert Schick, a.a.O., S. 245 f., vgl. auch Florian Edinger, Fraktion und Präsidium, in: Recht und Politik 1995, S. 81.

13 Vgl. Plenarprotokoll 13/1 vom 10. November 1994, S. 13. 
nahm ${ }^{14}$, obgleich das drohende Fehlen eines Vertreters der Linken im Bundestagspräsidium offenkundig war.

Auf der einen Seite fügten die Abgeordneten dem Ziel, dass jede Fraktion im Bundestagspräsidium vertreten sein soll, ihre eigene Lesart hinzu: Jede Fraktion ja, aber nicht mit jedem Kandidaten - ein Zusatz, der zu Missstimmung nicht nur bei den Linken führte. Auf der anderen Seite eröffnete das Plenum der Fraktion Die Linke damit die Möglichkeit zur Selbststilisierung: Sie konnte sich, wie vormals die PDS in vielfältigen Situationen des parlamentarischen und außerparlamentarischen Lebens, als von den anderen Parteien ausgegrenzt darstellen. Die Linke formulierte selbst, was ihr Zuwarten bis zu einem Alternativvorschlag bedeuten sollte: Protest. ${ }^{15}$ Beide Seiten, das Plenum und die Fraktion, trugen somit jeweils ihren Teil zu einem strukturellen Konflikt bei, über dessen Inhalte eine andere Debatte zu führen gewesen wäre und der die demokratietheoretischen wie parlamentsrechtlichen Fragestellungen unberechtigt in den Hintergrund verwies: Zu Beginn der 13. Wahlperiode einen Vertretungsanspruch jeder Fraktion in der GO-BT festzuschreiben, reichte nicht aus, um auch noch für den quantitativ und politisch stärker fragmentierten 16. Bundestag die Repräsentation jeder Fraktion im Präsidium sicherzustellen.

Der Konflikt führte nach einiger Zeit zu Bewegung bei beiden Akteuren: Am 27. März 2006 nominierte die Linke auf Vorschlag des unterlegenen Bisky Petra Pau als Bundestagsvizepräsidentin. Daraufhin wählte das Plenum diese am 7. April 2006 mit 385 Ja-Stimmen, 138 Nein-Stimmen und 58 Enthaltungen. ${ }^{16}$ Petra Pau erhielt damit noch immer ersichtlich weniger Ja-Stimmen und mehr Nein-Stimmen als jeder andere erfolgreiche Kandidat, erfüllte aber das Quorum deutlich. Überdies änderte der Bundestag seine GO dahingehend, dass einem in den ersten beiden Wahlgängen erfolglosen Einzelbewerber im dritten Wahlgang die Mehrheit der abgegebenen Stimmen zur Wahl ausreicht ( $\$ 2$ Abs. 2 S. 3 GO-BT 2006) sowie weitere Wahlgänge danach (nur) nach Vereinbarung im Ältestenrat zulässig sind und bei Neuvorschlägen erneut in den Wahlgang einzutreten ist ( $\$ 2$ Abs. 2 S. 3 GOBT 2006).

\section{I 2 GO-BT: Präsenzanspruch der Fraktionen versus Wahlrecht des Plenums}

Die GO-BT 2005 bestimmte in $\$ 2$ über die Wahl des Präsidenten und der Stellvertreter:

„(1) Der Bundestag wählt mit verdeckten Stimmzetteln (\$ 49) in besonderen Wahlhandlungen den Präsidenten und seine Stellvertreter für die Dauer einer Wahlperiode. Jede Fraktion des Deutschen Bundestages ist durch mindestens einen Vizepräsidenten oder eine Vizepräsidentin im Präsidium vertreten.

(2) Gewählt ist, wer die Stimmen der Mehrheit der Mitglieder des Bundestages erhält. Ergibt sich im ersten Wahlgang keine Mehrheit, so können für einen zweiten Wahlgang neue Bewerber vorgeschlagen werden. Ergibt sich auch dann keine Mehrheit der Stimmen der Mitglieder des Bundestages, so kommen die beiden Anwärter mit den höchsten Stimmenzahlen

14 Relativ gesehen, stimmten im 1. Wahlgang 38 v.H., im 2. Wahlgang 50 v.H., im 3. Wahlgang 46 v.H. und im 4. Wahlgang lediglich 42 v.H. für Lothar Bisky.

15 Vgl. die Presseerklärung der Fraktion Die Linke vom 28. März 2006, http://www.linksfraktion. de/nachricht.php?artikel=1456109870 (letzter Aufruf 24. Oktober 2007).

16 http://www.bundestag.de/aktuell/archiv/2006/wahl_pau/ (letzter Aufruf 24. Oktober 2007). 
in die engere Wahl. Bei Stimmengleichheit entscheidet das Los durch die Hand des amtierenden Präsidenten."

Der Passus war unglücklich formuliert: $₫ 2$ Abs. 1 S. 2 bestimmte im Indikativ mit der Vertretung jeder Fraktion im Präsidium ein politisch gewünschtes Ergebnis. Rechtlich ist diese Formulierung einer Faktizität als unbedingter Anspruch jeder Fraktion auf mindestens ein Vizepräsidentenamt zu deuten: Eine bloße Absichtserklärung ohne Bindungswirkung hätte anders lauten müssen („soll ... vertreten sein“). Gleichzeitig verlangte Abs. 2 eine - ergebnisoffene - Wahl, dies in Übereinstimmung sowohl mit der Freiheit der Abgeordneten gemäß Art. 38 Abs. 1 S. 2 GG als auch mit der Vorgabe des Art. 40 Abs. 1 S. 1 GG, wonach der Bundestag seinen Präsidenten sowie dessen Stellvertreter „wähle“. Eine Kollisionslösung für den Fall, dass die Wahlen nicht so ausfallen, dass der Anspruch einer jeden Fraktion auf Präsenz im Präsidium des Bundestages erfüllt ist, sah die GO-BT 2005 nicht vor. Sie stellte sich selbst damit eine Falle: die parallele Normierung eines Ergebnisses und eines Verfahrens, ohne dass das Verfahren zwingend zu dem Ergebnis führt.

Bei näherer Betrachtung handelte es sich bei der fehlenden Kollisionslösung um die Vorwegnahme der erwünschten, a priori feststehenden Wahlausgänge, die der Bundestag als Geschäftsordnungsgeber vor Augen hatte, denn die GO-BT 2005 sah einen dritten Wahlgang nur in Form der Stichwahl zwischen den zwei Bestplatzierten des zweiten Wahlgangs vor. Hieraus folgt, dass der Geschäftsordnungsgeber allein dann einen dritten Wahlgang für erforderlich hielt, wenn sich die Stimmen zuvor auf mehrere Bewerber verteilt und eben deshalb keiner von ihnen die absolute Mehrheit erreicht hatte. Bei der Verabschiedung der GO-BT ging der Bundestag der jahrzehntelangen Übung gemäß also davon aus, dass konkurrenzlose Kandidaten spätestens im zweiten Wahlgang das Plazet des Plenums erhalten. Das korrespondiert mit der Beobachtung, dass die von den Fraktionen benannten Kandidaten in der Regel auch gewählt wurden. ${ }^{17}$ Parlamentarisches Gewohnheitsrecht war hieraus jedoch mangels der erforderlichen aufgrund innerer Überzeugung gepflogenen und gleichmäßigen Übung über einen längeren Zeitraum ${ }^{18}$ nicht geworden - ein Grundsitz wurde jeder Fraktion erst zu Beginn der 13. Wahlperiode eingeräumt ${ }^{19}$. Bei der regelmäßigen Wahl der von den Fraktionen vorgeschlagenen Kandidaten handelt es sich daher lediglich um einen Parlamentsbrauch ${ }^{20}$ mit Appellfunktion, aber ohne rechtliche Verbind-

17 Vgl. Hans Troßmann, a.a.O., $\$ 2$ Rn. 2.4; Karl-Hans Rothaug, Die Leitungskompetenz des Bundestagspräsidenten, Kassel 1979, S. 167 spricht gar von der „Besetzungsberechtigung“ der Fraktionen.

18 Der Argumentation von Marcus Schuldei, Die Pairing-Vereinbarung, Berlin 1997, S. 71 f. mit weiteren Nachweisen, derzufolge an die Konstanz der Übung keine allzu hohen Anforderungen zu stellen sind, weil gemäß $\$ 126$ GO-BT selbst geschriebene GO-Regeln für den Einzelfall anders interpretiert, geändert oder außer Kraft gesetzt werden können, ist dabei nicht zu folgen: Hierfür bedarf es einer Zweidrittelmehrheit des Plenums, das von ihm als richtig oder auch nur zweckmäßig empfundene vermeintlich gewohnheitsrechtliche Regeln also auch im Einzelfall durchsetzen könnte. An die Konstanz der Übung sind daher die allgemeinen Anforderungen zu stellen.

19 Kandidaten der Grünen vor Beginn der 13. Legislaturperiode wurden eben gerade nicht in das Bundestagspräsidium gewählt, weshalb die Regelung eingeführt wurde, vgl. die Argumentationen im Protokoll, a.a.O.

$20 \mathrm{Zu}$ Differenzierung und Terminologie von parlamentarischem Gewohnheitsrecht und Parlamentsbrauch vgl. Thomas Schwerin, a.a.O., S. 260 ff.; Mathias Kühnreich, Das Selbstorganisationsrecht des Bundestages unter besonderer Berücksichtigung des Hauptstadtbeschlusses, Berlin 1997, S. 144 ff.; Marcus Schuldei, a.a.O., S. 72, Fn. 130; ferner Siegfried Magiera, in: Michael 
lichkeit. Allenfalls der Anspruch der größten Fraktion, den Bundestagspräsidenten zu stellen, mag gewohnheitsrechtlich anerkannt sein ${ }^{21}$, wobei auch dies vor dem Hintergrund der Freiheit des Mandats der Parlamentarier kritisch zu sehen ist. ${ }^{22}$

Wählt das Plenum den von einer Fraktion vorgeschlagenen konkurrenzlosen Einzelkandidaten entgegen dem Parlamentsbrauch nicht, entsteht ${ }^{23}$ ein Spannungsverhältnis zwischen der sowohl in $\$ 2$ Abs. 1 Satz 1 GO-BT als auch in Art. 40 Abs. 1 Satz 1 GG vorgesehenen Wahl und der in $\$ 2$ Abs. 1 Satz 2 GO-BT normierten Präsenz jeder Fraktion im Präsidium. Es stellt sich daher die Frage, ob im Kollisionsfall dem Verfahren der Wahl oder dem Ziel der Vertretung der Vorrang einzuräumen und die GO-BT entsprechend auszulegen und anzuwenden ist.

Dafür, das Ziel der Vertretung stärker zu gewichten, spricht zunächst das Telos des vorgesehenen Ergebnisses. Die GO selbst spricht davon, jede Fraktion sei durch mindestens einen Vizepräsidenten im Präsidium „vertreten“. Mindestens und daher ganz sicher ist in diesem Zusammenhang mit „Vertretung“ gemeint, dass jede Fraktion durch die Anwesenheit eines Stellvertreters im Bundestagspräsidium präsent ist. Diese Präsenz ist, weil nicht nur mit Sitz, sondern auch mit Stimme ausgestattet, mehr als physische Anwesenheit; sie ist Ausdruck einer aktiven, gestaltenden Rolle. Es begegnet jedoch Bedenken, die Vertretung im Sinne von Repräsentation zu deuten: Denn gerade staatsorganisationsrechtlich ist der Topos der Repräsentation den Abgeordneten vorbehalten, die gemäß Art. 38 Abs. 1 Satz 2 GG, jeder für sich, das ganze Wahlvolk repräsentieren. ${ }^{24}$ Die Fraktionen hingegen sind an Parteimitgliedschaften orientierte Zusammenschlüsse von Abgeordneten, sie bilden insofern nur einen Bruchteil, eben eine Fraktion, des Plenums. Ein Verständnis, nach dem die Vizepräsidenten die Fraktionen in dem Sinne repräsentieren wie dies die Funktion der Abgeordneten für das Volk ist, billigte der Parteimitgliedschaft als Organisationsmerkmal der Fraktionen einen zu hohen Stellenwert in der Nähe des von Gerhard Leibholz vertretenen „Parteienstaats“ zu. ${ }^{25}$ Die Parteien wirken an der Willensbildung des Volkes gemäß Art. 21 Abs. 1 S. 1 GG lediglich mit und benennen etwa Kandidaten für die Parlamentswahlen;

Sachs, Grundgesetz-Kommentar, 3. Auflage, München 2003, Art. 40 Rn. 23 und Martin Morlok, in: Horst Dreier, Grundgesetz-Kommentar, Band II, Tübingen 1998, Art. 40 Rn. 8; kritisch zur Diktion: Helmuth Schulze-Fielitz, Parlamentsbrauch, Gewohnheitsrecht, Observanz in: HansPeter Schneider / Wolfgang Zeh (Hrsg.), a.a.O., \$11 Rn. 11 ff.; Gerald Kretschmer, Geschäftsordnungen deutscher Volksvertretungen, in: Hans-Peter Schneider / Wolfgang Zeh (Hrsg.), a.a.O., S. $\$ 9$ Rn. $62 \mathrm{ff}$.

21 Vgl. Thomas Schwerin, a.a.O., S. 262 mit weiteren Nachweisen; Helmuth Schulze-Fielitz, a.a.O.; in diese Richtung auch Wolfgang Zeh, in: Josef Isensee, Paul Kirchhof (Hrsg.), Handbuch des Staatsrechts der Bundesrepublik Deutschland, Band II, Heidelberg 1987, $\$ 42$ Rn. 29 und Joseph Bücker, a.a.O., $\$ 27$ Rn. 2.

22 Die größte Fraktion muss nicht zwangsläufig auch der Regierungsmehrheit angehören. Es ist daher jedenfalls vorstellbar, dass die Regierungsmehrheit den Vertreter der größten Fraktion nicht zum Bundestagspräsidenten wählt. Ein gewohnheitsrechtlicher Anspruch der Fraktion beziehungsweise des Betroffenen auf Wahl durch den Bundestag müsste nach Ansicht des Autors an der verfassungsrechtlich garantierten Freiheit des Mandats scheitern. Wenn auch ohne diese Begründung im Ergebnis ähnlich Karl-Heinz Kleinschnittger, Die rechtliche Stellung des Bundestagspräsidenten, Diss., Bielefeld 1963, S. 19 f.; vgl. auch Rupert Schick, a.a.O., S. $27 \mathrm{ff}$.

23 Dies auch unbeschadet der in diesem Beitrag diskutierten Änderung.

24 Freilich spricht auch Art. 38 GG davon, die Abgeordneten seien „Vertreter“ des ganzen Volkes.

25 Vgl. hierzu und im Folgenden: Jörn Ipsen, in: Michael Sachs, a.a.O., Art. 21 Rn 12 ff. und Martin Morlok, a.a.O., Art. 21 Rn. 156 ff. 
innerhalb des Parlaments selbst verfügen sie als solche jedoch über keinen Repräsentationsanspruch, hier ist das gemäß Art. 38 Abs. 1 Satz 2 GG freie Mandat entscheidend. Insofern hat die GO-BT mit der „Vertretung“ aller Fraktionen im Bundestagspräsidium einen passenden Begriff gewählt: Etwas weniger als Repräsentation, aber etwas mehr als bloße Präsenz, die aber immer auch gemeint ist.

Dass jede Fraktion mindestens einen Vizepräsidenten stellt, entspringt somit dem Gedanken der möglichst umfassenden Teilhabe des fraktionierten Plenums an der Bildung des Präsidiums. ${ }^{26}$ Zwar wird zuvörderst der Präsident für den Bundestag als Gesamtheit tätig ${ }^{27}$, das den Präsidenten umfassende Präsidium ist mithin nicht als Kollegialorgan zu interpretieren, dessen Mitglieder jeder für sich präsidentielle Aufgaben und Befugnisse hat. ${ }^{28}$ Vielmehr ist es vor allem ein Beratungsorgan des Präsidenten, das in besonderer Weise dem Ausgleich für das Gesamtparlament verpflichtet ist. Diese Funktion kann nur durch die Präsenz aller Fraktionen realisiert werden ${ }^{29}$, die daher wichtiger als deren relativ zum Plenum proportionale Berücksichtigung in diesem verhältnismäßig kleinen Gremium ist. ${ }^{30}$ Daher war es ein im Ergebnis richtiger Schritt des 13. Bundestages, jeder Fraktion einen Grundsitz ${ }^{31}$ im Präsidium einzuräumen, wobei nicht vergessen werden darf, dass hier auch parteipolitischer Zwist eine erhebliche Rolle spielte. ${ }^{32}$

Für den Vorrang der Vertretung jeder Fraktion gegenüber dem Wahlverfahren spricht zudem, dass praktisch nur für Kandidaten von Oppositionsfraktionen die Gefahr besteht, trotz Abwesenheit von Konkurrenz keine absolute Mehrheit zu bekommen. Die Wahl des Bundestagspräsidiums steht am Anfang einer Wahlperiode, wenn die zukünftige Regierungsmehrheit sich gerade gefunden hat, mithin auch und gerade bei knappen Mehrheits-

26 Der Parlamentarische Geschäftsführer der CDU/CSU-Fraktion Norbert Röttgen sprach in der konstituierenden Sitzung des 16. Bundestages sogar ausdrücklich von Repräsentation, vgl. Protokoll, 6 B, während Jörg van Essen (FDP) vorsichtiger formulierte, jede Fraktion solle sich im Präsidium „,wiederfinden“, vgl. Protokoll 7 D. Vgl. Siegfried Magiera, a.a.O., Art. 40 Rn. 5; Martin Morlok, a.a.O., Art. 40 Rn. 25.

27 Statt vieler Siegfried Magiera, a.a.O., Art. 40 Rn. 8. Zu den Aufgaben des Präsidenten siehe ausführlich Karl-Hans Rothaug, a.a.O., passim; Karl-Heinz Kleinschnittger, a.a.O., passim; Wolfgang Zeh, a.a.O., $\$ 42$ Rn. 30 ff. und Thomas Wilrich, Der Bundestagspräsident, in: DÖV 2002, S. $152 \mathrm{ff}$., passim.

28 Vgl. $\$ 7$ GO-BT, der den Präsidenten, nicht das Präsidium mit Aufgaben und Befugnissen ausstattet. Siehe auch Thomas Wilrich, a.a.O., S. $157 \mathrm{ff}$.

29 Vgl. Karl-Hans Rothaug, a.a.O., S. 171, der das Spannungsverhältnis zwischen dem Anspruch der Unparteilichkeit und der Fraktionszugehörigkeit des Bundestagspräsidenten anspricht.

30 Zum Proporzgedanken siehe Florian Edinger, Wahl und Besetzung parlamentarischer Gremien, Berlin 1992, S. $172 \mathrm{ff}$.

31 Der Begriff „Grundmandat“ erscheint hier insofern verfehlt, als dass die Fraktionen „ihre“ Vizepräsidenten gerade nicht wie etwa Ausschussmitglieder mandatieren, also mit dem Auftrag versehen, ihre Positionen durchzusetzen. Vgl. zur Frage des Grundmandats bei der Besetzung parlamentarischer Gremien Florian Edinger, a.a.O. (1992), S. 272 ff., für das Präsidium ausdrücklich S. 304 ff., allerdings vor der entsprechenden Änderung der GO-BT.

$32 \mathrm{Zu}$ Beginn der 13. Wahlperiode verweigerte sich die Union dem Wunsch der SPD, wie bisher zwei Vizepräsidenten zu stellen, lehnte den Antrag ab, das Präsidium zu vergrößern und wählte die Kandidatin der Grünen, Antje Vollmer, mit. 1998 hatte die SPD diesen Affront nicht vergessen und verhinderte ihrerseits, dass die Union einen zweiten Vizepräsidentenposten bekam. Vgl. Michael F. Feldkamp, Datenhandbuch zur Geschichte des Deutschen Bundestages 1994 bis 2003. Ergänzungsband, Baden-Baden 2005, S. 235; Stenographische Protokolle, 14. WP, A. Sitzung, S. $10 \mathrm{ff}$. 
verhältnissen abweichende Stimmen aus der Mehrheit also praktisch kaum zu erwarten sind. Eine Mehrheit zu Gunsten eines Kandidaten der Opposition ist indes nicht in gleicher Weise sicher. Aus Art. 38 Abs. 1 Satz 2 GG folgt jedoch, dass jeder Abgeordnete grundsätzlich die gleichen parlamentarischen Mitwirkungsbefugnisse innehat. Abgeordnete der Opposition, der in einer parlamentarischen Demokratie auch eine politische Integrationsfunktion $^{33}$ zukommt, dürfen keinesfalls diskriminiert werden. ${ }^{34}$ Daher haben die Fraktionen grundsätzlich ein gleiches Recht auf Zugang zu parlamentarischen Gremien. ${ }^{35}$ Dies gilt für das Bundestagspräsidium als Organ, das den Ausgleich für das gesamte Parlament leisten soll, in noch höherem Maße als etwa für Ausschüsse, von denen die Plenumsmehrheit zu Recht erwarten darf, dass sie Schlussabstimmungen auch im Sinne ihrer politischen Mehrheitsmeinung vorbereiten.

Zunächst sprechen also erhebliche Argumente dafür, dem vorgesehenen Ergebnis Vorrang vor dem vorgeschriebenen Verfahren einzuräumen. Vor diesem Hintergrund hätte die GO-BT 2005 etwa dahingehend ausgelegt werden können, dass sich der Vertretungsanspruch der Fraktionen nach zwei erfolglosen Wahlgängen in einem Entsendungsrecht realisiert. Dem steht jedoch der unbedingte Geltungsvorrang der Verfassung entgegen: Art. 40 Abs. 1 Satz 1 GG, der die Wahl des Bundestagspräsidiums und damit einen politischen Entscheidungsakt des Plenums ${ }^{36}$ fordert, ist somit eine Grenze der Geschäftsordnungsautonomie ${ }^{37}$, die der Nachfolgesatz konstituiert. Die GO-BT 2005 durfte also nicht dahingehend ausgelegt werden, den Fraktionen gleichsam ein Entsendungsrecht zuzubilligen. Der von Bundestagspräsident Norbert Lammert eingeschlagene Weg eines dritten (und vierten) Wahlgangs war der Formulierung der GO-BT zwar nicht direkt zu entnehmen, stimmte aber mit den verfassungsrechtlichen Vorgaben überein.

\section{Die Änderung der GO-BT}

Der Bundestag fasste daraufhin am 21. September 2006 einstimmig den Beschluss, $\$ 2$ Abs. 2 GO-BT nach Satz 2 wie folgt zu ergänzen:

„Ergibt sich auch dann [nach dem zweiten Wahlgang] keine Mehrheit der Stimmen der Mitglieder des Bundestages, findet ein dritter Wahlgang statt. Bei nur einem Bewerber ist dieser gewählt, wenn er die Mehrheit der abgegebenen Stimmen auf sich vereinigt. Bei mehreren Bewerbern kommen die beiden Bewerber mit den höchsten Stimmenzahlen in die engere Wahl; gewählt ist, wer die meisten Stimmen auf sich vereinigt. Bei Stimmengleichheit entscheidet das Los durch die Hand des amtierenden Präsidenten."

Und er fügte $\$ 2$ GO-BT einen neuen Absatz 3 an:

„(3) Weitere Wahlgänge mit einem im dritten Wahlgang erfolglosen Bewerber sind nur nach Vereinbarung im Ältestenrat zulässig. Werden nach erfolglosem Ablauf des Verfahrens nach

33 Vgl. hierzu Stephan Haberland, Die verfassungsrechtliche Bedeutung der Opposition nach dem Grundgesetz, Berlin 1995, S. 45 f.

$34 \mathrm{Vgl}$. ebenda, S. $69 \mathrm{f}$.

35 Ebenda, S. 71 f.; auch für die institutionalisierte Abgeordnetengruppe sieht dies Reinhold Kassing, Das Recht der Abgeordnetengruppe, Berlin 1988, S. 36, so (explizit für den Ältestenrat).

36 „Ein Parlament muß frei sein zu bestimmen, wer sein Präsident sein soll.“ (Carlo Schmid, zitiert nach: Rupert Schick, a.a.O., S. 28).

37 Vgl. Martin Morlok, a.a.O., Art. 40 Rn. 7. 
Absatz 2 neue Bewerber vorgeschlagen, ist neu in das Wahlverfahren gemäß Absatz 2 einzutreten."

Der Bundestag entschied sich somit für eine abgestufte Reaktion für den Fall, dass ein Einzelbewerber im ersten und zweiten Wahlgang erfolglos bleibt: Zunächst senkte er das Quorum für den dritten Wahlgang von der Kanzler- auf die einfache Mehrheit ab. Sodann eröffnete er vorbehaltlich einer Vereinbarung im Ältestenrat den Weg zu weiteren Wahlgängen, verpflichtete sich selbst jedoch darauf, erneut in das Wahlverfahren einzutreten, wenn nach einem erfolglosen dritten Wahlgang neue Bewerber vorgeschlagen werden.

\section{Würdigung}

Die Analyse des vom Bundestag eingeschlagenen Wegs zeigt zunächst, dass er lediglich eine von verschiedenen Möglichkeiten gewählt hat, dem strukturellen Konflikt zu begegnen, von denen zunächst beispielhaft zwei aufgezeigt und ihrerseits gewürdigt werden sollen.

(1) Interfraktionelle Vereinbarung: In den ersten neun Wahlperioden wich das Parlament aufgrund einer interfraktionellen Vereinbarung von dem in der GO-BT vorgesehenen Wahlverfahren ab und wählte alle Vizepräsidenten offen und in einem Wahlgang en bloc. ${ }^{38}$ Dieser Weg macht es zwar unwahrscheinlicher, aber nicht unmöglich, dass einzelne Kandidaten nicht gewählt werden; lediglich die Fraktionsdisziplin erlegt den Abgeordneten auf, sich an interfraktionelle Vereinbarungen zu halten. Möchten sie die Freiheit ihres Mandats betonen, mögen sie gemäß $₫ 47$ GO-BT die Teilung der Frage beantragen. Überdies entziehen derartige interfraktionelle Vereinbarungen dem Plenum die Möglichkeit, den Grad seiner Zustimmung zu den einzelnen Kandidaten auszudrücken. ${ }^{39}$

(2) Absenken des Quorums: In Anlehnung an die Praxis des dritten und vierten Wahlgangs für Bisky hätte das Quorum für weitere Wahlgänge noch weiter abgesenkt werden können. Betrüge es für den dritten Wahlgang beispielsweise nur ein Viertel, benötigte das Plenum eine „Sperrmajorität“ von drei Vierteln seiner Mitglieder, um einen Kandidaten aus dem Präsidium fernzuhalten. Hierdurch würde die Präsenz aller Fraktionen im Präsidium wahrscheinlicher gemacht, ohne vom Wahlverfahren abzurücken. Die Wahlvorgabe des Art. 40 Abs. 1 S. 1 GG wäre gewahrt, weil dieser kein bestimmtes Quorum vorgibt. Indes würde dem Parlament ein Vizepräsident zugemutet, der nicht einmal auf eine relative Mehrheit bei seiner Wahl verweisen kann, dessen Gesamtvertretungsanspruch daher geschmälert wäre. Zudem schlösse auch dieses Verfahren nicht gänzlich aus, dass konkurrenzlose Bewerber durchfallen. Schließlich müsste das Quorum stets höher sein als die jeweilige konkrete Fraktionsstärke, weil sich das Verfahren sonst in ein - strukturell systemwidriges - Entsendungsrecht umwandeln würde.

Die vom Bundestag getroffene Regelung stärkt im Gegensatz zu diesen Optionen dessen Selbstorganisationsrecht. Zu begrüßen ist auch, dass sie strikt die Wahlvorgabe des Art. 40

38 Rupert Schick, a.a.O., S. 32 f.

39 Auch von Lothar Bisky abgesehen, schwankte die Zustimmung des Plenums bei den Wahlen zum Präsidium des 16. Bundestages erheblich: Der Präsident Norbert Lammert (CDU) erhielt 564 von 606 abgegebenen Stimmen, das waren 93 v.H., sein Amtsvorgänger Wolfgang Thierse (SPD) hingegen nur 417 von 605 abgegeben Stimmen, also 69 v.H., eine sowohl absolut als auch relativ deutlich geringere Zustimmung. 
Abs. 1 S. 1 GG beachtet und den Gesamtvertretungsanspruch des Bundestagspräsidiums insofern stärkt, als sich jegliches Wahlergebnis auf Entscheidungen der Mehrheit und / oder des Ältestenrates zurückführen lässt. Die Problematik liegt indes darin, den strukturellen Konflikt zwischen der Ergebnis- und der Verfahrensvorgabe nicht zu lösen und lediglich einen ersten Schritt zur Prozeduralisierung seiner Bewältigung festzuhalten. Indem für einen dritten Wahlgang eines konkurrenzlosen Bewerbers das Quorum auf die einfache Mehrheit abgesenkt wird, wird dessen Wahl zwar wahrscheinlicher; indessen wäre etwa gerade die Causa Bisky hierdurch nicht befriedigend beendet worden. Das weitere Prozedere dem Ältestenrat zu überlassen, enthebt das amtierende Bundestagspräsidium zwar der Last von ad hoc-Entscheidungen, dürfte jedoch zu Einzelfallabsprachen führen, die dem oben genannten Gedanken einer gleichberechtigten Teilhabe an den innerparlamentarischen Gestaltungs- und Mitwirkungsrechten nicht ideal entspricht. Schließlich ist hierdurch nicht sichergestellt, dass der Vizepräsidentenposten wirklich besetzt wird.

\section{Eigener Regelungsvorschlag: Vorschlagsobliegenheit der Fraktion}

Als Alternative zu der verabschiedeten Regelung ist daran zu denken, der vorschlagenden Fraktion nach zwei erfolglosen Wahlgängen sowohl zu ermöglichen als auch aufzugeben, dem Plenum einen Alternativkandidaten für die Stichwahl vorzuschlagen, andernfalls sie den Anspruch auf Vertretung im Präsidium verlöre.

Dieses Verfahren knüpft an zwei Regelungsgedanken bereits in der GO-BT 2005 an: zum einen an die Möglichkeit des Plenums, nach einem ersten erfolglosen Wahlgang weitere Kandidaten vorzuschlagen ( $\$ 2$ Abs. 2 S. 2 GO-BT), zum anderen an die Stichwahl aus Satz 3, bei der die einfache Mehrheit zur Wahl ausreicht. Bundestagspräsident Lammert hat dieses gegenüber den ersten beiden Wahlgängen modifizierte Quorum bereits auf den dritten und vierten Wahlgang für Bisky angewandt, was seine Begründung stichhaltig macht, er habe das Quorum aus dem „Gesamtkontext der Regelungen“ 40 entnommen: Die Wahlgänge drei und vier verliefen quasi so, als sei ein virtueller Gegenkandidat mit der Bezeichnung „einstweilen unbesetzt“ gegen Bisky angetreten. Der hier vertretene Ansatz billigt im Falle des erfolglosen Einzelkandidaten der betroffenen Fraktion auch noch für den dritten Wahlgang ein Vorschlagsrecht zu, beschränkt dieses aber auf eben diese Fraktion. Mit anderen Worten: Nur die betroffene Fraktion darf für die Stichwahl einen Alternativkandidaten nachnominieren. Unterlässt sie dies und unterliegt der Einzelkandidat auch im dritten Wahlgang, hat sie ihren Anspruch, im Präsidium vertreten zu sein, verwirkt.

Das Verfahren hat den Nachteil, die betroffene Fraktion in der Auswahl ihres Kandidaten insofern einzuschränken, als sie sich nicht länger auf einen einzigen Kandidaten festlegen kann, sondern dem Plenum eine Alternative vorschlagen muss. Indes normiert selbst der „fraktionsfreundliche“ $\$ 2$ Abs. 1 Satz 1 GO-BT nicht den Anspruch einer Fraktion, durch einen bestimmten Kandidaten, sondern lediglich überhaupt im Präsidium vertreten zu sein. Aus dem Selbstorganisationsrecht des Bundestages gemäß Art. 40 Abs. 1 S. 1 GG folgt in Verbindung mit dem Gebot der Wahl zu dessen Präsidium die Mitwirkungsobliegenheit der Fraktion, nicht auf einem einzigen Kandidaten zu beharren, sondern eine 
(Aus-)Wahl durch das Plenum zu ermöglichen. Kommt die Fraktion dieser Obliegenheit nicht nach, ist es aufgrund des verfassungsrechtlich begründeten Vorrangs des Wahlverfahrens gegenüber dem Präsenzergebnis hinzunehmen, dass sie dort nicht vertreten ist.

Die Vorteile dieses Vorschlags bestehen darin, sich zunächst bruchlos in das bisherige Wahlverfahren der GO-BT 2005 einzufügen. Sodann ist eine Konsultation des Ältestenrates - mit ungewissem Ausgang - nicht erforderlich; das weitere Verfahren ist für jeden Abgeordneten transparent. Insbesondere ist hiernach das Verfahren definitiv nach drei Wahlgängen beendet, eine unbefriedigende und letztlich das Bundestagspräsidium schwächende Schwebesituation kann somit nicht ungewollt ${ }^{41}$ eintreten.

Allerdings geht mit diesem Verfahren eine partielle Auswahlbeschränkung des Plenums einher: Im dritten Wahlgang kann es sich nurmehr zwischen zwei Kandidaten einer Fraktion entscheiden. Art. 40 Abs. 1 S. 1 GG steht dem jedoch nicht entgegen: Unbenommen bleibt die Möglichkeit des Plenums, sowohl bereits für den ersten Wahlgang als auch gemäß $\$ 2$ Abs. 2 Satz 2 GO-BT für den zweiten Wahlgang andere Bewerber vorzuschlagen, ob diese nun aus der betroffenen Fraktion stammen oder nicht. Wenngleich es nicht der Zielvorgabe der Präsenz aller Fraktionen im Bundestagspräsidium entspricht, ist es durchaus parlamentarische Praxis, Gegenkandidaten zu nominieren. ${ }^{42}$ Somit hat das Plenum die Möglichkeit, Einfluss auf die Auswahl der beiden Stichwahlkandidaten zu nehmen, was die Auswahlentscheidung nicht allein in die Hand der Fraktionen legt: Fällt der konkurrenzlose Einzelkandidat einer Fraktion im ersten Wahlgang durch, hat es das Plenum in der Hand, entweder weitere Bewerber für den zweiten Wahlgang vorzuschlagen und damit Einfluss auf die Auswahl in der Stichwahl zu nehmen oder den Kandidaten allein in einem zweiten Wahlgang zu wählen oder durchfallen zu lassen. Nur für den Fall, dass er zum zweiten Mal keine Mehrheit erzielt, hat allein die betroffene Fraktion ein Nachnominierungsrecht für einen Alternativkandidaten. Die bei der Stichwahl auftretende personelle Auswahlbeschränkung des Plenums zu Gunsten der Sicherheit der Fraktion, im Präsidium vertreten zu sein, ist also vorhersehbar und rührt maßgeblich daher, dass das Plenum das Vorschlagsrecht für den zweiten Wahlgang nicht ausgeübt hat. Dieses Vorschlagsrecht ist nach Maßgabe des Selbstorganisationsgebots aus Art. 40 GG allerdings notwendig von ihm selbst auszuüben. Überlässt das Plenum die Auswahlentscheidung zu Gunsten des in $\$ 2$ Abs. 1 Satz 2 GOBT selbst gesetzten Ziels der Präsenz jeder Fraktion den jeweiligen Fraktionen, obwohl es anders agieren könnte, liegt hierin keine Beschränkung seines Wahlrechts aus Art. 40 Abs. 1 Satz $1 \mathrm{GG}^{43}$ : Das Plenum hat das Wahlrecht dann bereits - negativ - ausgeübt.

41 Sollte das Plenum die Schwebesituation jedoch wollen, um etwa in interfraktionellen Absprachen eine Lösung herbeizuführen, kann es den dritten Wahlgang bis zum Abschluss dieser aussetzen.

42 So schlugen zu Beginn der 13. Wahlperiode zunächst Manfred Müller (Berlin, PDS) die Abgeordnete Dagmar Enkelmann von der PDS alternativ zu Hans Klein (München) von der CDU/CSU vor, sodann Peter Struck (SPD) die Abgeordnete Anke Fuchs von der SPD zunächst alternativ zu Antje Vollmer von Bündnis 90/Die Grünen und nach deren Wahlgewinn alternativ zu Burkhard Hirsch von der FDP vor, vgl. Plenarprotokoll vom 10. November 1994, 14 D, 15 D, 16 B. Zu Beginn der 14. Wahlperiode schlug die Fraktion der CDU/CSU die Abgeordnete Michaela Geiger (CSU) alternativ zu Petra Bläss (PDS) vor, vgl. Plenarprotokoll vom 26. Oktober 1998, 18 A. Die Entscheidungen, die jeweiligen Gegenkandidaten nicht zu wählen, entsprangen in vollem Umfang der Wahlautonomie des Plenums.

43 Das Verfahren der Stichwahl unter zwei Bewerbern, im Fall mehrerer Erstbewerber der beiden Bestplatzierten, als solches begegnet keinen wahlverfahrensrechtlichen Bedenken, da es die Letztentscheidung stufenweise konkretisiert. Ergänzend sei darauf hingewiesen, dass das verfassungs- 
An dem hier vertretenen Verfahren ist somit einzig der Umstand neu, dass die betroffene Fraktion, und zwar als einzige, einen Alternativkandidaten für die Stichwahl nachnominieren und so ihren Vertretungsanspruch im Präsidium sichern kann.

Schließlich wird das Spannungsverhältnis zwischen Ziel- und Verfahrensvorgabe, das auch die GO-BT 2006 nicht vollständig beseitigt, sicher aufgelöst: Schlägt die bislang erfolglose Fraktion zum dritten Wahlgang einen Alternativkandidaten vor, kann sie sich sicher sein, anschließend im Präsidium vertreten zu sein, denn einer ihrer beiden Kandidaten wird die Stichwahl gewinnen. Das Wahl- und damit auch Auswahlrecht verbleibt jedoch bis einschließlich des zweiten Wahlgangs allein in der Hand des Plenums.

Dieser Vorschlag könnte durch Einfügung folgender Passage ${ }^{44}$ in $\$ 2$ Abs. 2 nach Satz 4 GO-BT 2005 verwirklicht werden:

„Stand lediglich ein Kandidat zur Wahl, kann die Fraktion, die durch ihn vertreten sein will, für den dritten Wahlgang einen weiteren Kandidaten vorschlagen. Macht sie von dieser Möglichkeit keinen Gebrauch, verliert sie den Anspruch aus $\$ 2$ Abs. 1 Satz 2.“

Somit hätte der Bundestag als Repräsentant des Souveräns stets das gewichtigste Wort bei der Wahl zu seinem Präsidium. Gleichwohl könnte sich das Plenum durch schlichtes NichtHandeln, nämlich durch die Nicht-Nominierung weiterer Kandidaten für den zweiten Wahlgang, seiner beiden Ziele versichern, jede Fraktion im Präsidium vertreten lassen zu sein und zugleich eine Wahl im Sinne des Art. 40 Abs. 1 S. 1 GG durchzuführen.

Trotz dieser feineren Abstimmung zwischen dem Wahlrecht des Plenums und dem Präsenzanspruch der Fraktionen wird deutlich: Die Problematik, die sich anhand der Kandidatur Biskys gezeigt hat, ist eine primär politische, die im Konfliktfall rechtlich allenfalls befriedet, aber nicht unbedingt zu allseitiger Befriedigung gelöst werden kann. Das verfassungsrechtliche Wahlrecht geht dem lediglich geschäftsordnungsrechtlichen Präsenzanspruch vor - letzterer kann lediglich verfahrenstechnisch aufgewertet werden, wozu dieser Beitrag anregen möchte. Dem Plenum kann jedoch nicht der Vertreter einer Fraktion als Vizepräsident aufgezwungen werden, den dieses partout nicht wünscht. Es ist eine Frage der Politischen Kultur, im Konfliktfall in einen konstruktiven Dialog einzutreten. Nur falls dieser scheitert, kann dem Geschäftsordnungsrecht klarstellende Funktion zukommen. Die vorgeschlagene Modifikation gibt der Geschäftsordnung hinsichtlich des erfolglosen Einzelkandidaten die erforderliche Eindeutigkeit und Ergebnissicherheit.

rechtlich normierte Wahlverfahren für den Bundespräsidenten in Art. 54 Abs. 6 GG nach zwei Wahlgängen ohne absolute Mehrheit der gesetzlichen Mitglieder der Bundesversammlung die einfache Mehrheit ausreichen lässt, sogar ohne dass sich zuvor die Auswahl auf die beiden Bestplatzierten verkürzt hätte: Bei einer entsprechend großen Anzahl von Bewerbern kann also sogar der Bundespräsident gegen den Willen der deutlichen Mehrheit der Bundesversammlung gewählt werden.

44 Bei gleichzeitiger Rücknahme der Änderungen vom 26. September 2006. 